\title{
Efficacy of Propanil against Weeds in Direct Seeded Rice and Its Effect on Succeding Crop
}

\author{
Y. M. Ramesha ${ }^{1^{\star}}$, Manjunatha Bhanuvally², Ashok Kumar Gaddi ${ }^{2}$, \\ D. Krishamurthy ${ }^{1}$ and M. R. Umesh ${ }^{1}$ \\ ${ }^{1}$ Department of Agronomy, University of Agricultural Sciences, Raichur, Karnataka, India. \\ ${ }^{2}$ Department of Soil Science and Agricultural Chemistry, University of Agricultural Sciences, Raichur,
} Karnataka, India.

\section{Authors' contributions}

This work was carried out in collaboration between all authors. Author YMR designed the study, performed the statistical analysis, wrote the protocol and wrote the first draft of the manuscript. Authors MB and AKG managed the analyses of the study. Authors DK and MRU managed the literature searches. All authors read and approved the final manuscript.

Article Information

DOI: $10.9734 /$ IJPSS/2017/33555

Editor(s):

(1) Radim Vacha, Deputy Director of Research and Development, Research Institute for Soil and Water Conservation,

Czech Republic.

Reviewers:

(1) Bilal Ahmad Lone, Shere Kashmir University of Agricultural Sciences and Technology, India. (2) Kowthar Gad Aly El-Rokiek, National Research Centre, Egypt Complete Peer review History: http://www.sciencedomain.org/review-history/19964

Original Research Article

Received $20^{\text {th }}$ April 2017

Accepted $11^{\text {th }}$ May 2017

Published $10^{\text {th }}$ July 2017

\section{ABSTRACT}

Aims: To assess the efficacy of propanil against weeds in direct seeded rice.

Place and Duration: A field study was conducted during Kharif 2015 and summer 2016, at Agricultural Research Station, Dhadesugur, University of Agricultural Sciences, Raichur, Karnataka, India.

Methodology: An investigation comprises of eight treatments and replicated thrice. The weeds which were dominant in trials field are Echinichloa sp. Panicum repens, Leptochloa chinensis, Brachiaria mutica, Digitaria sanguinalis among grasses, Eclipta alba, Ludwigia parviflora and Commelina communis as broad leaf weeds and Cyperus sp. as sedge.

Results: Application of Propanil 80\% DF @ $4 \mathrm{~kg}$ a.i./ha and twice hand weeded check at 30 and 45 days after sowing found significantly $(p=0.05)$ superior over the application of Propanil $80 \%$ DF @ 3 $\mathrm{kg}$ a.i./ha and rest of the treatments in controlling the weeds in direct seeded rice and increases the grain yield of rice without any phytotoxic effect.

Conclusion: Propanil 80\% DF @ $3 \mathrm{~kg}$ a.i./ha could be recommended for post-emergence application at 10 to 15 days after sowing of paddy crop to achieve effective control of weeds. 
Keywords: Dry weight of weeds; weed control efficiency; Propanil; grain yield.

\section{INTRODUCTION}

Cereals are the most important part of our diet throughout the world and thus, play major role in our food security. Among cereals, rice has been staple food for more than 60 per cent of the world population, providing energy for about $40 \%$ of the world population where every third person on earth consumes rice every day in one form or other [1]. Therefore, crop paddy (Oryza sativa L.) is an important crop which is extensively grown in tropical and subtropical regions of the world. There are several reasons for its low productivity but the losses due to weeds are one of the most important. More than one third of the total loss $(33 \%)$ is caused by weeds alone [2]. Weeds are most severe and widespread biological constraints to crop production in India. Weeds are responsible for heavy yield losses in paddy, to the extent of complete crop failure under severe infestation conditions. Irrespective of the method of paddy establishment, weeds are a major impediment to paddy production due to their ability to compete for resources. In general, weeds problem in transplanted paddy is lower than that of direct seeded paddy because of puddling and stagnation of water in transplanted paddy during early growth stage of crop. But in some cases where continuous standing water cannot be maintained particularly for the first 45 days, weed infestation in transplanted paddy also may be as high as direct seeded paddy. Weeds can reduce the grain yield of dry-seeded paddy (DSR) by $75.8 \%$, wet seeded paddy (WSR) by $70.6 \%$ and transplanted paddy (TPR) by $62.6 \%$. Weeds by virtue of their high adaptability and faster growth dominate the crop habitat and reduce the yield potential [3]. Therefore, the present investigation was undertaken to study the effect of early post emergent herbicide for control of major weeds in direct seeded rice.

\section{MATERIALS AND METHODS}

\subsection{Background of the Study}

A field study was taken during Kharif-2015 and Summer-2016 at Agricultural Research Station, Dhadesugur. The soil of the experimental site was medium deep black and neutral in $\mathrm{pH}$ (8.04), EC $(0.47 \mathrm{ds} / \mathrm{m})$, medium in organic carbon content $(0.41 \%)$, low in nitrogen $(189 \mathrm{~kg} / \mathrm{ha})$, medium in phosphorus (58.5 kg/ha) and potassium $(287.5 \mathrm{~kg} / \mathrm{ha})$.

\subsection{Treatment Details}

This experiment was comprises of eight treatments viz., $\mathbf{T}_{1}$ : Propanil 80\% DF @ $1.0 \mathrm{~kg}$ a.i/ha, $\mathbf{T}_{2}$ : Propanil 80\% DF @ $2.0 \mathrm{~kg}$ a.i/ha, $\mathbf{T}_{3}$ : Propanil 80\% DF @ $3.0 \mathrm{~kg}$ a.i/ha, $\mathbf{T}_{4}$ : Propanil $80 \%$ DF @ $4.0 \mathrm{~kg}$ a.i/ha, T $_{5}$ : Oxyfluorfen $23.5 \%$ EC @ $240 \mathrm{~g}$ a.i./ha, $\mathbf{T}_{6}$ : Cyhalofop butyl 10\% EC @ $80 \mathrm{~g}$ a.i./ha, $\mathbf{T}_{7}$ : Hand weeding and $\mathbf{T}_{8}$ : Weedy check.

\subsection{Trial Details}

This study was laid out with randomized complete block design and replicated thrice with a plot size of $6 \mathrm{~m}$ in length and $4 \mathrm{~m}$ in width. Land is prepared well with harrowing and brings in to fine tilth condition. Dry paddy seeds were sown at a spacing of $21 \mathrm{~cm} \times 15 \mathrm{~cm}$ in first week of July during Kharif 2015 and first week of December in summer 2016. Immediately after sowing, irrigation was given and later irrigation was given as and when crop requires. Recommended dose of fertilizer (150:75:75 kg NPK/ha) was applied uniformly in three equal splits (Application of $50 \% \mathrm{~N}$ through urea, $100 \%$ $\mathrm{P}_{2} \mathrm{O}_{5}$ through DAP and $50 \%$ potash through MOP at first split. Application of $25 \% \mathrm{~N}$ through urea and $25 \%$ potash through MOP at second split and application of remaining $25 \% \mathrm{~N}$ through urea and $25 \%$ potash through MOP at third split). Other agronomic and plant protection measures were adopted as recommended during the crop growth.

\subsection{Application of Herbicides and Efficacy Evaluation}

Herbicides were sprayed as per the treatments (at 10-15 days after sowing of crop or at 2-3 leaf stage of weeds) using a Knapsack sprayer fitted with a flat nozzle at a spray volume of $500 \mathrm{l} / \mathrm{ha}$. The efficacy of different treatments on weeds was evaluated at crop maturity. Quadrates $(0.25$ $\mathrm{m}^{2}$ ) were placed in each plot at random to determine the weed density. Weed seedlings within these quadrates were counted and the efficacy of weed control treatments was evaluated by comparing the density with the untreated control. Weeds were cut at ground level, washed with tap water, oven dried at $70^{\circ} \mathrm{C}$ for 48 hours and then weighed for dry matter. The weed control efficiency was calculated using the formula as follows [4]. 


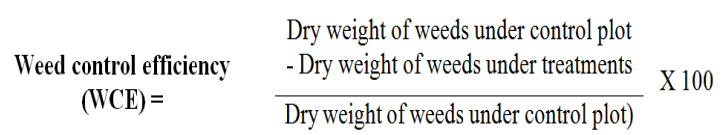

\subsection{Data Collection and Economics}

After harvest and threshing of crop, grain yield was recorded in net plot wise and converted to grain yield per hectare basis. The cost of inputs that were prevailing at the time of their use was considered for working out the economics of various treatments. Net return per hectare was calculated by deducting the cost of cultivation from gross returns per hectare, gross returns was calculated by using the total income obtained from grain and straw yield of rice and the benefit cost ratio was worked out as follows.

$$
\text { Benefit cost ratio }=\frac{\text { Gross returns }(\mathrm{Rs} / \mathrm{ha})}{\text { vost of cultivation }(\mathrm{Rs} / \mathrm{ha})}
$$

\subsection{Succeeding Crop}

To see the impact of herbicides on succeeding crop, the black gram crop was sown after harvesting of the paddy from the herbicides treated plots and the data recorded on germination of seed and impact on crop growth and development viz. Leaf injury on tips and Leaf surface, Wilting, Vein clearing, Necrosis, Epinasty, Hyponasty, stunted growth etc. after 7, 15 and 21 days after germination (DAG). The data from in each year analysed separately.

\subsection{Data Analysis}

MSTAT was used for statistical analysis of data and means were separated using critical difference (CD) at $p=0.05$. The data on weeds were transformed by square root transformation before being subjected to ANOVA [5].

\section{RESULTS AND DISCUSSION}

\subsection{Weed Flora in the Experimental Field}

The weeds which were dominant in trials field are Echinichloa sp. Panicum repens, Leptochloa chinensis, Brachiaria mutica, Digitaria sanguinalis among grasses, Eclipta alba, Ludwigia parviflora and Commelina communis as broad leaf weeds and Cyperus sp. as sedge.

\subsection{Effect of Propanil on Weed Density}

The data on weed density is presented in Tables 1 and 2. Results revealed that, all the weed management treatments were significantly $(p=0.05)$ reduced weeds populations as compared to Oxyfluorfen 23.5\% EC @ 240 g a.i./ha and weedy check in Kharif 2015 and Summer 2016 when observed at 45 DAS in direct seeded rice. Among the herbicidal treatments, application of Propanil 80\% DF @ 4 $\mathrm{kg}$ a.i./ha was recorded significantly $(p=0.05)$ lowest weeds population and which was onpar with the application of Propanil 80\% DF @ $3 \mathrm{~kg}$ a.i./ha and twice hand weeded check at 45 DAS. Further, application of Propanil 80\% DF @ 2 kg a.i./ha was the next treatment in terms of controlling weeds after Oxyfluorfen 23.5\% EC @ $240 \mathrm{~g}$ a.i./ha. These results are conformity with the findings of Amarasinghe and Marambe [6].

\subsection{Effect of Propanil on Dry Weight of Weeds}

The data on dry weight of weeds is presented in Table 3. Results observed that, application of Propanil 80\% DF @ 4 kg a.i/ha, 3 kg a.i./ha and twice hand weeded check recorded significantly higher dry weight of weeds over the application of Propanil 80\% DF @ 2 kg a.i./ha and rest of the treatments except Oxyfluorfen 23.5\% EC @ 240 $g$ a.i./ha. Similarly, application of Propanil $80 \%$ DF @ 4, 3 and 2 kg a.i//ha doses were recorded least dry weight. These results are conformity with the findings of Abeysekera [7] stated that, application of tank mixture of quichlorac @ 50 $\mathrm{g} / \mathrm{ha}+$ propanil @ $1.08 \mathrm{~kg} / \mathrm{ha}$ controlled effectively the grassy weeds and recorded lower dry weight in wet seeded rice in mid country region of Srilanka. Whereas, higher dry weight of grassy weeds was observed in weedy check treatment.

\subsection{Effect of Propanil on Weed Control Efficiency (WCE)}

Results revealed that, all the weed management treatments are significantly recorded higher weed control efficiency as compared to weedy check in Kharif 2015 and Summer 2016 when observed at 45 DAS in direct seeded rice. Among the herbicidal treatments, application of Propanil 80\% DF @ 4 kg a.i/ha was recorded significantly higher weed control efficiency (85.2 and $86.52 \%$ during Kharif 2015 and summer 2016, respectively) and which was onpar with the application of propanil $80 \%$ DF @ 3 kg a.i./ha and twice hand weeded check over the rest of the treatments except Oxyfluorfen 23.5\% EC @ 240 g a.i./ha. Further, application of Propanil 80\% DF @ 4, 3 and $2 \mathrm{~kg}$ a.i/ha doses were recorded significantly least weed control efficiency. 
Ramesha et al.; IJPSS, 17(3): 1-7, 2017; Article no.IJPSS.33555

Table 1. Effect of weed control treatments on weed population (count $\left./ \mathrm{m}^{2}\right)$ in DSR at 45 DAS $\left(1^{\text {st }}\right.$ season-Kharif 2015)

\begin{tabular}{|c|c|c|c|c|c|c|c|c|c|}
\hline \multirow[t]{2}{*}{ Treatments } & \multicolumn{5}{|c|}{ Grasses } & \multicolumn{3}{|c|}{ Broad leaf weeds } & \multirow{2}{*}{$\begin{array}{l}\text { Sedges } \\
\text { Cyperus } \\
\text { sp. }\end{array}$} \\
\hline & $\begin{array}{l}\text { Echinichloa } \\
\text { sp. }\end{array}$ & $\begin{array}{l}\text { Panicum } \\
\text { repens }\end{array}$ & $\begin{array}{l}\text { Leptochloa } \\
\text { chinensis }\end{array}$ & $\begin{array}{l}\text { Brachiaria } \\
\text { mutica }\end{array}$ & $\begin{array}{l}\text { Digitaria } \\
\text { sanguinalis }\end{array}$ & $\begin{array}{l}\text { Eclipta } \\
\text { alba }\end{array}$ & $\begin{array}{l}\text { Ludwigia } \\
\text { parviflora }\end{array}$ & $\begin{array}{l}\text { Commelina } \\
\text { communis }\end{array}$ & \\
\hline $\mathrm{T}_{1}$ : Propanil 80\% DF @ $1.0 \mathrm{~kg}$ a.i/ha & $\begin{array}{l}2.00 \\
(1.73)\end{array}$ & $\begin{array}{l}1.33 \\
(1.53)\end{array}$ & $\begin{array}{l}1.33 \\
(1.53)\end{array}$ & $\begin{array}{l}1.00 \\
(1.41)\end{array}$ & $\begin{array}{l}1.67 \\
(1.63)\end{array}$ & $\begin{array}{l}1.33 \\
(1.53)\end{array}$ & $\begin{array}{l}2.00 \\
(1.73)\end{array}$ & $\begin{array}{l}3.30 \\
(2.07)\end{array}$ & $\begin{array}{l}7.67 \\
(2.94)\end{array}$ \\
\hline $\mathbf{T}_{2}$ : Propanil 80\% DF @ $2.0 \mathrm{~kg}$ a.i/ha & $\begin{array}{l}0.67 \\
(1.29)\end{array}$ & $\begin{array}{l}0.33 \\
(1.15)\end{array}$ & $\begin{array}{l}0.33 \\
(1.15)\end{array}$ & $\begin{array}{l}0.67 \\
(1.29)\end{array}$ & $\begin{array}{l}0.33 \\
(1.15)\end{array}$ & $\begin{array}{l}0.33 \\
(1.15)\end{array}$ & $\begin{array}{l}0.33 \\
(1.15)\end{array}$ & $\begin{array}{l}0.67 \\
(1.29)\end{array}$ & $\begin{array}{l}6.33 \\
(2.71)\end{array}$ \\
\hline $\mathbf{T}_{3}$ : Propanil 80\% DF @ $3.0 \mathrm{~kg}$ a.i/ha & $\begin{array}{l}0.33 \\
(1.15)\end{array}$ & $\begin{array}{l}0.00 \\
(1.00)\end{array}$ & $\begin{array}{l}0.00 \\
(1.00)\end{array}$ & $\begin{array}{l}0.33 \\
(1.15)\end{array}$ & $\begin{array}{l}0.00 \\
(1.00)\end{array}$ & $\begin{array}{l}0.00 \\
(1.00)\end{array}$ & $\begin{array}{l}0.00 \\
(1.00)\end{array}$ & $\begin{array}{l}1.00 \\
(1.41)\end{array}$ & $\begin{array}{l}5.67 \\
(2.58)\end{array}$ \\
\hline $\mathbf{T}_{4}:$ Propanil $80 \%$ DF @ $4.0 \mathrm{~kg}$ a.i/ha & $\begin{array}{l}0.00 \\
(1.00)\end{array}$ & $\begin{array}{l}0.33 \\
(1.15)\end{array}$ & $\begin{array}{l}0.00 \\
(1.00)\end{array}$ & $\begin{array}{l}0.00 \\
(1.00)\end{array}$ & $\begin{array}{l}0.33 \\
(1.15)\end{array}$ & $\begin{array}{l}0.00 \\
(1.00)\end{array}$ & $\begin{array}{l}0.33 \\
(1.15)\end{array}$ & $\begin{array}{l}0.67 \\
(1.29)\end{array}$ & $\begin{array}{l}6.33 \\
(2.71)\end{array}$ \\
\hline $\mathrm{T}_{5}:$ Oxyfluorfen $23.5 \%$ EC @ $240 \mathrm{~g}$ a.i./ha & $\begin{array}{l}6.33 \\
(2.71)\end{array}$ & $\begin{array}{l}4.33 \\
(2.31)\end{array}$ & $\begin{array}{l}3.33 \\
(2.08)\end{array}$ & $\begin{array}{l}4.33 \\
(2.31)\end{array}$ & $\begin{array}{l}3.33 \\
(2.08)\end{array}$ & $\begin{array}{l}1.00 \\
(1.41)\end{array}$ & $\begin{array}{l}3.67 \\
(2.16)\end{array}$ & $\begin{array}{l}2.33 \\
(1.82)\end{array}$ & $\begin{array}{l}4.33 \\
(2.31)\end{array}$ \\
\hline $\mathbf{T}_{6}$ : Cyhalofop butyl $10 \%$ EC @ $80 \mathrm{~g}$ a.i./ha & $\begin{array}{l}0.00 \\
(1.00)\end{array}$ & $\begin{array}{l}0.00 \\
(1.00)\end{array}$ & $\begin{array}{l}1.33 \\
(1.53)\end{array}$ & $\begin{array}{l}0.67 \\
(1.29)\end{array}$ & $\begin{array}{l}0.33 \\
(1.15)\end{array}$ & $\begin{array}{l}7.67 \\
(2.94)\end{array}$ & $\begin{array}{l}4.00 \\
(2.24)\end{array}$ & $\begin{array}{l}7.00 \\
(2.83)\end{array}$ & $\begin{array}{l}13.00 \\
(3.74)\end{array}$ \\
\hline $\mathbf{T}_{7}$ : Hand weeding & $\begin{array}{l}0.00 \\
(1.00)\end{array}$ & $\begin{array}{l}0.00 \\
(1.00)\end{array}$ & $\begin{array}{l}0.33 \\
(1.15)\end{array}$ & $\begin{array}{l}0.67 \\
(1.29)\end{array}$ & $\begin{array}{l}0.00 \\
(1.00)\end{array}$ & $\begin{array}{l}0.00 \\
(1.00)\end{array}$ & $\begin{array}{l}0.00 \\
(1.00)\end{array}$ & $\begin{array}{l}0.00 \\
(1.00)\end{array}$ & $\begin{array}{l}1.67 \\
(1.63)\end{array}$ \\
\hline $\mathrm{T}_{8}$ : Weedy check & $\begin{array}{l}11.33 \\
(3.51)\end{array}$ & $\begin{array}{l}3.33 \\
(2.08)\end{array}$ & $\begin{array}{l}5.67 \\
(2.58)\end{array}$ & $\begin{array}{l}7.33 \\
(2.89)\end{array}$ & $\begin{array}{l}4.00 \\
(2.24)\end{array}$ & $\begin{array}{l}7.00 \\
(2.83)\end{array}$ & $\begin{array}{l}4.33 \\
(2.31)\end{array}$ & $\begin{array}{l}6.67 \\
(2.77)\end{array}$ & $\begin{array}{l}12.67 \\
(3.70)\end{array}$ \\
\hline CD at $5 \%$ & 0.41 & 0.35 & 0.35 & 0.33 & 0.44 & 0.31 & 0.35 & 0.29 & 1.34 \\
\hline
\end{tabular}

Note: Figures in the parenthesis are square root transformed values (sq. root of $x+1$ ), DAS: Days after sowing 
Ramesha et al.; IJPSS, 17(3): 1-7, 2017; Article no.IJPSS.33555

Table 2. Effect of weed control treatments on weed population (count $\left./ \mathrm{m}^{2}\right)$ in DSR at 45 DAS $\left(2^{\text {nd }}\right.$ Season -summer 2016)

\begin{tabular}{|c|c|c|c|c|c|c|c|c|c|}
\hline \multirow[t]{2}{*}{ Treatments } & \multicolumn{5}{|c|}{ Grasses } & \multicolumn{3}{|c|}{ Broad leaf weeds } & \multirow{2}{*}{$\begin{array}{l}\text { Sedges } \\
\text { Cyperus } \\
\text { sp. }\end{array}$} \\
\hline & $\begin{array}{l}\text { Echinichloa } \\
\text { sp. }\end{array}$ & $\begin{array}{l}\text { Panicum } \\
\text { repens }\end{array}$ & $\begin{array}{l}\text { Leptochloa } \\
\text { chinensis }\end{array}$ & $\begin{array}{l}\text { Brachiaria } \\
\text { mutica }\end{array}$ & $\begin{array}{l}\text { Digitaria } \\
\text { sanguinalis }\end{array}$ & $\begin{array}{l}\text { Eclipta } \\
\text { alba }\end{array}$ & $\begin{array}{l}\text { Ludwigia } \\
\text { parviflora }\end{array}$ & $\begin{array}{l}\text { Commelina } \\
\text { communis }\end{array}$ & \\
\hline $\mathbf{T}_{1}$ : Propanil 80\% DF @ $1.0 \mathrm{~kg}$ a.i/ha & $\begin{array}{l}3.33 \\
(2.08)\end{array}$ & $\begin{array}{l}1.00 \\
(1.41)\end{array}$ & $\begin{array}{l}3.67 \\
(2.16)\end{array}$ & $\begin{array}{l}1.67 \\
(1.63)\end{array}$ & $\begin{array}{l}0.67 \\
(1.29)\end{array}$ & $\begin{array}{l}1.67 \\
(1.63)\end{array}$ & $\begin{array}{l}4.33 \\
(2.31)\end{array}$ & $\begin{array}{l}1.67 \\
(1.63)\end{array}$ & $\begin{array}{l}5.67 \\
(2.58)\end{array}$ \\
\hline $\mathrm{T}_{2}$ : Propanil 80\% DF @ $2.0 \mathrm{~kg}$ a.i/ha & $\begin{array}{l}1.00 \\
(1.41)\end{array}$ & $\begin{array}{l}0.00 \\
(1.00)\end{array}$ & $\begin{array}{l}1.33 \\
(1.53)\end{array}$ & $\begin{array}{l}0.67 \\
(1.29)\end{array}$ & $\begin{array}{l}0.33 \\
(1.15)\end{array}$ & $\begin{array}{l}1.00 \\
(1.41)\end{array}$ & $\begin{array}{l}1.00 \\
(1.41)\end{array}$ & $\begin{array}{l}0.67 \\
(1.29)\end{array}$ & $\begin{array}{l}4.00 \\
(2.24)\end{array}$ \\
\hline $\mathrm{T}_{3}$ : Propanil 80\% DF @ $3.0 \mathrm{~kg}$ a.i/ha & $\begin{array}{l}0.00 \\
(1.00)\end{array}$ & $\begin{array}{l}0.00 \\
(1.00)\end{array}$ & $\begin{array}{l}0.00 \\
(1.00)\end{array}$ & $\begin{array}{l}0.00 \\
(1.00)\end{array}$ & $\begin{array}{l}0.00 \\
(1.00)\end{array}$ & $\begin{array}{l}1.00 \\
(1.41)\end{array}$ & $\begin{array}{l}1.00 \\
(1.41)\end{array}$ & $\begin{array}{l}1.00 \\
(1.41)\end{array}$ & $\begin{array}{l}3.67 \\
(2.16)\end{array}$ \\
\hline $\mathbf{T}_{4}$ : Propanil 80\% DF @ $4.0 \mathrm{~kg}$ a.i/ha & $\begin{array}{l}0.00 \\
(1.00)\end{array}$ & $\begin{array}{l}0.00 \\
(1.00)\end{array}$ & $\begin{array}{l}0.00 \\
(1.00)\end{array}$ & $\begin{array}{l}0.00 \\
(1.00)\end{array}$ & $\begin{array}{l}0.00 \\
(1.00)\end{array}$ & $\begin{array}{l}0.33 \\
(1.15)\end{array}$ & $\begin{array}{l}0.67 \\
(1.29)\end{array}$ & $\begin{array}{l}1.00 \\
(1.41)\end{array}$ & $\begin{array}{l}3.33 \\
(2.08)\end{array}$ \\
\hline $\mathrm{T}_{5}:$ Oxyfluorfen $23.5 \%$ EC @ $240 \mathrm{~g}$ a.i./ha & $\begin{array}{l}4.67 \\
(2.38)\end{array}$ & $\begin{array}{l}1.67 \\
(1.63)\end{array}$ & $\begin{array}{l}3.30 \\
(2.07)\end{array}$ & $\begin{array}{l}3.00 \\
(2.00)\end{array}$ & $\begin{array}{l}1.67 \\
(1.63)\end{array}$ & $\begin{array}{l}2.00 \\
(1.73)\end{array}$ & $\begin{array}{l}2.33 \\
(1.82)\end{array}$ & $\begin{array}{l}2.00 \\
(1.73)\end{array}$ & $\begin{array}{l}5.33 \\
(2.52)\end{array}$ \\
\hline $\mathrm{T}_{6}:$ Cyhalofop butyl $10 \%$ EC @ $80 \mathrm{~g}$ a.i./ha & $\begin{array}{l}0.00 \\
(1.00)\end{array}$ & $\begin{array}{l}0.00 \\
(1.00)\end{array}$ & $\begin{array}{l}1.00 \\
(1.41)\end{array}$ & $\begin{array}{l}0.33 \\
(1.15)\end{array}$ & $\begin{array}{l}0.00 \\
(1.00)\end{array}$ & $\begin{array}{l}4.67 \\
(2.38)\end{array}$ & $\begin{array}{l}10.33 \\
(3.37)\end{array}$ & $\begin{array}{l}5.33 \\
(2.52)\end{array}$ & $\begin{array}{l}9.00 \\
(3.16)\end{array}$ \\
\hline $\mathbf{T}_{7}$ : Hand weeding & $\begin{array}{l}0.00 \\
(1.00)\end{array}$ & $\begin{array}{l}0.33 \\
(1.15)\end{array}$ & $\begin{array}{l}0.00 \\
(1.00)\end{array}$ & $\begin{array}{l}0.00 \\
(1.00)\end{array}$ & $\begin{array}{l}0.33 \\
(1.15)\end{array}$ & $\begin{array}{l}0.00 \\
(1.00)\end{array}$ & $\begin{array}{l}0.00 \\
(1.00)\end{array}$ & $\begin{array}{l}1.00 \\
(1.41)\end{array}$ & $\begin{array}{l}1.00 \\
(1.41)\end{array}$ \\
\hline $\mathbf{T}_{8}$ : Weedy check & $\begin{array}{l}8.33 \\
(3.05)\end{array}$ & $\begin{array}{l}2.67 \\
(1.92)\end{array}$ & $\begin{array}{l}7.33 \\
(2.89)\end{array}$ & $\begin{array}{l}4.67 \\
(2.38)\end{array}$ & $\begin{array}{l}2.00 \\
(1.73)\end{array}$ & $\begin{array}{l}5.67 \\
(2.58)\end{array}$ & $\begin{array}{l}9.67 \\
(3.27)\end{array}$ & $\begin{array}{l}4.67 \\
(2.38)\end{array}$ & $\begin{array}{l}8.33 \\
(3.05)\end{array}$ \\
\hline CD at $5 \%$ & 0.51 & 0.34 & 0.63 & 0.40 & 0.23 & 0.25 & 0.48 & 0.20 & 0.34 \\
\hline
\end{tabular}

Table 3. Assessment of weeds dry weights $\left(\mathrm{g} / \mathrm{m}^{2}\right)$ from different herbicidal treatments in DSR at 45 DAS

\begin{tabular}{|c|c|c|c|c|c|c|c|c|c|c|}
\hline \multirow[t]{3}{*}{ Treatments } & \multicolumn{10}{|c|}{ Weed dry weight $\left(\mathrm{g} / \mathrm{m}^{2}\right)$} \\
\hline & \multicolumn{2}{|c|}{ Grasses } & \multicolumn{2}{|c|}{ BLW } & \multicolumn{2}{|c|}{ Sedges } & \multicolumn{2}{|c|}{ Total } & \multicolumn{2}{|c|}{ WCE (\%) } \\
\hline & 2015 & 2016 & 2015 & 2016 & 2015 & 2016 & 2015 & 2016 & 2015 & 2016 \\
\hline $\mathbf{T}_{1}$ : Propanil 80\% DF @ $1.0 \mathrm{~kg}$ a.i/ha & 6.34 & 6.88 & 4.96 & 4.70 & 5.41 & 3.75 & 16.72 & 15.34 & 62.93 & 57.48 \\
\hline $\mathbf{T}_{2}$ : Propanil 80\% DF @ $2.0 \mathrm{~kg}$ a.i/ha & 3.26 & 2.71 & 1.36 & 1.67 & 4.30 & 2.50 & 8.93 & 6.88 & 82.42 & 80.92 \\
\hline T $_{3}$ : Propanil 80\% DF @ $3.0 \mathrm{~kg}$ a.i/ha & 2.03 & 1.04 & 0.90 & 2.08 & 4.08 & 1.97 & 7.02 & 5.10 & 84.44 & 85.87 \\
\hline $\mathrm{T}_{4}$ : Propanil $80 \%$ DF @ $4.0 \mathrm{~kg}$ a.i/ha & 1.58 & 1.03 & 1.11 & 1.75 & 3.98 & 2.08 & 6.67 & 4.86 & 85.20 & 86.52 \\
\hline $\mathrm{T}_{5}:$ Oxyflourfen $23.5 \%$ EC @ $240 \mathrm{~g}$ a.i./ha & 15.63 & 9.78 & 5.90 & 4.38 & 2.94 & 3.33 & 24.47 & 17.49 & 45.74 & 51.51 \\
\hline $\mathrm{T}_{6}:$ Cyhalofop butyl $10 \%$ EC @ $80 \mathrm{~g}$ a.i./ha & 3.85 & 1.46 & 12.70 & 15.03 & 8.84 & 8.57 & 25.38 & 25.06 & 43.72 & 30.51 \\
\hline$T_{7}$ : Hand weeding & 1.81 & 2.72 & 1.24 & 0.75 & 2.04 & 1.17 & 5.09 & 4.64 & 88.72 & 87.13 \\
\hline $\mathrm{T}_{8}$ : Weedy check & 21.76 & 17.52 & 14.50 & 12.30 & 8.84 & 6.26 & 45.10 & 36.07 & -- & -- \\
\hline CD at $5 \%$ & 1.43 & 1.68 & 1.77 & 2.04 & 1.88 & 1.10 & 3.24 & 4.58 & -- & -- \\
\hline
\end{tabular}


Table 4. Effect of Propanil $80 \%$ DF on the grain yield of direct seeded rice

\begin{tabular}{|c|c|c|c|c|}
\hline \multirow[t]{2}{*}{ Treatments } & \multicolumn{2}{|c|}{ Grain Yield $(q / h a)^{\star}$} & \multicolumn{2}{|c|}{ C:B ratio } \\
\hline & 2015 & 2016 & 2015 & 2016 \\
\hline $\mathrm{T}_{1}$ : Propanil 80\% DF @ $1.0 \mathrm{~kg}$ a.i/ha & 57.38 & 52.3 & 1:1.15 & $1: 1.35$ \\
\hline $\mathrm{T}_{2}$ : Propanil 80\% DF @ $2.0 \mathrm{~kg}$ a.i/ha & 60.88 & 58.12 & $1: 1.98$ & $1: 1.84$ \\
\hline $\mathrm{T}_{3}$ : Propanil 80\% DF @ $3.0 \mathrm{~kg}$ a.i/ha & 62.48 & 58.90 & $1: 2.23$ & $1: 2.41$ \\
\hline $\mathrm{T}_{4}$ : Propanil $80 \%$ DF @ $4.0 \mathrm{~kg}$ a.i/ha & 62.12 & 59.12 & $1: 2.19$ & $1: 2.34$ \\
\hline $\mathrm{T}_{5}$ : Oxyfluorfen $23.5 \%$ EC @ $240 \mathrm{~g}$ a.i./ha & 56.61 & 49.43 & 1:0.92 & 1:1.05 \\
\hline $\mathrm{T}_{6}:$ Cyhalofop butyl $10 \% \mathrm{EC} @ 80 \mathrm{~g}$ a.i./ha & 55.35 & 50.12 & 1:1.05 & $1: 1.33$ \\
\hline$T_{7}:$ Hand weeding & 60.21 & 58.11 & $1: 2.00$ & $1: 2.33$ \\
\hline $\mathrm{T}_{8}:$ Weedy check & 51.67 & 45.62 & $1: 0.68$ & $1: 0.82$ \\
\hline$C D(P=0.05)$ & 4.10 & 5.41 & - & - \\
\hline
\end{tabular}

${ }^{*}$ Mean of 3 replications

Table 5. Phytotoxicity effect on growth parameters of succeeding crop black gram as influenced by the application of Propanil 80\% DF (Mean data of 2015 and 2016)

\begin{tabular}{|c|c|c|c|c|}
\hline \multirow[t]{2}{*}{ Treatments } & \multicolumn{3}{|c|}{ Phytotoxic effect (\%)* } & \multirow{2}{*}{$\begin{array}{l}\text { Germination } \\
\text { percent }\end{array}$} \\
\hline & 7 DAG & 15 DAG & 21 DAG & \\
\hline $\mathrm{T}_{1}$ : Propanil $80 \%$ DF @ $1.0 \mathrm{~kg}$ a.i/ha & 0.0 & 0.0 & 0.0 & 93.0 \\
\hline $\mathrm{T}_{2}$ : Propanil $80 \%$ DF @ $2.0 \mathrm{~kg}$ a.i/ha & 0.0 & 0.0 & 0.0 & 92.0 \\
\hline $\mathrm{T}_{3}$ : Propanil 80\% DF @ $3.0 \mathrm{~kg}$ a.i/ha & 0.0 & 0.0 & 0.0 & 93.6 \\
\hline $\mathrm{T}_{4}$ : Propanil $80 \%$ DF @ $4.0 \mathrm{~kg}$ a.i/ha & 0.0 & 0.0 & 0.0 & 92.0 \\
\hline $\mathrm{T}_{5}:$ Oxyfluorfen $23.5 \%$ EC @ $240 \mathrm{~g}$ a.i./ha & 0.0 & 0.0 & 0.0 & 91.6 \\
\hline $\mathrm{T}_{6}:$ Cyhalofop butyl $10 \%$ EC @ $80 \mathrm{~g}$ a.i./ha & 0.0 & 0.0 & 0.0 & 92.6 \\
\hline$T_{7}:$ Untreated & 0.0 & 0.0 & 0.0 & 93.6 \\
\hline
\end{tabular}

These results are conformity with the findings of Amarasinghe et al. [8] stated that, application of quichlorac@500 g/ha recorded higher weed control efficiency in wet seeded rice in mid country region of Srilanka. Similarly, lower weed control efficiency was noticed in weedy check treatment (Table 3).

\subsection{Grain Yield and Economics of Direct Seeded Rice}

Among the weed management treatments, Hand weeding at 15 and 45 days after sowing gave significantly higher grain yield over weedy check. However, application of Propanil 80\% DF @ 4 kg a.i/ha was at par with its lower dose i.e. Propanil 80\% DF @ $3 \mathrm{~kg}$ a.i./ha, found to be significantly superior and on par with recorded higher grain yield followed by twice hand weeding at 15 and 45 days after sowing. Moreover, maximum cost benefit ratio was observed in plots treated with Propanil $80 \%$ DF along with twice hand weeded check (Table 4). These results are conformity with the findings of Seema, et al. [9] stated that, higher grain yield of aerobic rice was recorded in weed control treatments over the un-weeded treatment.

\subsection{Effect of Herbicides on Succeeding Crop}

The phytotoxicity effect on succeeding black gram in terms of leaf necrosis, chlorosis or wilting was observed at 7,15 and 21 days after germination (DAG) at all dosages of Propanil $80 \%$ DF and other herbicides including untreated control. Results indicated that, there was no phytotoxicity effect (rating 0 ) noticed in all the plots in both the season (Table 5). Further there was no impact on germination of black gram seed which was sown after harvesting of paddy crop from Propanil $80 \%$ DF treated plot in both the season.

\section{CONCLUSION}

Results says that, application of Propanil $80 \%$ DF @ $3 \mathrm{~kg}$ a.i./ha could be recommended for post-emergence application at 10 to 15 days after sowing of paddy crop to achieve effective control of Echinochloa spp. (E. colona, $E$. crusgalli), Panicum repens, Leptochloa chinensis, Brachiaria mutica, Digitaria sanguinalis, Eclipta alba, Ludwigia parviflora, Commelina communis and Cyperus sp. Further, 
it produces higher grain yield and benefit cost ratio due to effective control of weeds in direct seeded rice.

\section{COMPETING INTERESTS}

Authors have declared that no competing interests exist.

\section{REFERENCES}

1. Datta SK, Khushi GS. Improving rice to meet food and nutrient needs: Biotechnological approaches. Journal of Crop Production. 2002;6:229-247.

2. Verma SK, Singh SB, Meena RN, Prasad SK, Meena RS, Gaurav. A review of weed management in India: The need of new directions for sustainable agriculture. The Bioscan. 2015;10(1):253-263.

3. Singh VP, Singh $G$, Singh $M$. Effect of fenoxaprop-pethyl on transplanted rice and associated weeds. Indian Journal of Weed Science. 2004;36(3\&4):190-192.

4. Tawaha AM, Turk MA, Maghaireh GA. Response of barley to herbicide versus mechanical weed control under semi arid conditions. Journal of Agronomy and Crop Science. 2002;188:106-112.

5. Gomez KA, Gomez AA. Statistical procedures for agricultural research (2 ed.). John Wiley and Sons, New York. 1984;680.

6. Amarasinghe L, Marambe B. Trends in weed control of rice cultivation in Sri Lanka. Proceedings of Multi-disciplinary International Conference. University of Peradeniya, Srilanka. 1998;1-12 (Supplement)

7. Abeysekera A. Current status of weed control in rice in Sri Lanka. Proceedings of the $17^{\text {th }}$ Asian Pacific Weed Science Conference, 22-27 November, Thailand. 1999;174-18.

8. Amarasinghe, Marambe LB, Rajpakse RPAD. Effect of quinchlorac on weed control and productivity of wet seeded rice in the mid region of Sri Lanka. Sri Lankan Journal of Agricultural Sciences. 1999;36: 24-34.

9. Seema, Krishna M, and Devi MTT. Effect of nitrogen and weed management on nutrient uptake by weeds under direct seeded aerobic rice. The Bioscan. 2014;9(2):535-537.

(c) 2017 Ramesha et al.; This is an Open Access article distributed under the terms of the Creative Commons Attribution License (http://creativecommons.org/licenses/by/4.0), which permits unrestricted use, distribution, and reproduction in any medium, provided the original work is properly cited.

Peer-review history:

The peer review history for this paper can be accessed here: http://sciencedomain.org/review-history/19964 